\title{
Minimally invasive stereotactic puncture and thrombolysis therapy improves long-term outcome after acute intracerebral hemorrhage
}

\author{
Houguang Zhou $\cdot$ Yu Zhang $\cdot$ Ling Liu $\cdot$ Yanyan Huang $\cdot$ Yuping Tang $\cdot$ \\ Jingjing Su $\cdot$ Wei Hua $\cdot$ Xu Han $\cdot$ Jianzhong Xue $\cdot$ Qiang Dong
}

Received: 25 October 2010/Revised: 10 December 2010/Accepted: 3 January 2011/Published online: 22 February 2011

(C) The Author(s) 2011. This article is published with open access at Springerlink.com

\begin{abstract}
The purpose of this study was to judge the clinical value of minimally invasive stereotactic puncture and thrombolysis therapy (MISPTT) for acute intracerebral hemorrhage (ICH). A randomized control clinical trial was undertaken. According to the enrollment criteria, 122 acute ICH cases were analyzed, of which 64 cases received MISPTT (MISPTT group, MG) and 58 cases received conventional craniotomy (CC group, CG). The Glasgow coma scale (GCS) scores, postoperative complications (PC), and rebleeding incidences were compared. Moreover, 1 year postoperation, the long-term outcomes of patients with regard to hematoma volume (HV) $<50 \mathrm{~mL}$ and $\mathrm{HV}$ $\geq 50 \mathrm{~mL}$ were judged, respectively, by the Glasgow
\end{abstract}

H. Zhou and Y. Zhang have contributed equally to the work

H. Zhou $\cdot$ Y. Tang $\cdot$ J. Su $\cdot$ X. Han $\cdot$ Q. Dong $(\bowtie)$ Department of Neurology, Huashan Hospital, Fudan University, 12\# Middle WuLuMuQi Rd, Shanghai 200040, China e-mail: qdong@ @ shmu.edu.cn

Y. Zhang $\cdot$ Y. Huang

Department of Geriatrics, Huashan Hospital, Fudan University,

12\# Middle WuLuMuQi Rd, Shanghai 200040, China

L. Liu

Department of Neurology, Jinling Hospital, Nanjing University

School of Medicine, 305\# East Zhongshan Rd,

Nanjing 210002, China

W. Hua

Department of Neurosurgery, Huashan Hospital,

Fudan University, 12\# Middle WuLuMuQi Rd,

Shanghai 200040, China

J. Xue

Department of Neurology, Affiliated ChangShu Hospital,

Yangzhou University, 68\# South HaiYu Rd,

Changshu 215500, China outcome scale (GOS), Barthel index (BI), modified Rankin Scale (mRS), and case fatality (CF). MG patients showed obvious amelioration in GCS score compared with that of CG patients. The total incidence of PC in MG decreased compared with that of CG. The incidences of rebleeding in MG and CG were 9.4 and $17.2 \%$, respectively $(P=0.243$ ). There were no obvious differences between the CFs of MG and CG (17.2 and 25.9\%, respectively, $P=0.199)$. The GOS, BI, and mRS representing long-term outcome for both $\mathrm{HV}<50 \mathrm{~mL}$ and $\mathrm{HV} \geq 50 \mathrm{~mL}$ in $\mathrm{MG}$ were ameliorated significantly greater than that in CG patients (all $P<0.05$ ). These data suggest that there are advantages with MISPTT not only in trauma and safety, but the MISPTT group had fewer complications and a trend toward improved shortterm and long-term outcomes.

Keywords Acute intracerebral hemorrhage - Minimally invasive stereotactic puncture and thrombolysis therapy . Conventional craniotomy · Urokinase

\section{Introduction}

Spontaneous intracerebral hemorrhage (ICH) comprises $8-30 \%$ of all stroke victims, depending on regional and ethnic differences, and is a devastating form of stroke with the high mortality twofold to sixfold higher than that for ischemic stroke [1] and a 1-year survival rate less than $50 \%$ [2]. Hitherto, morbidity and mortality following $\mathrm{ICH}$ remain the highest among all forms of cerebrovascular diseases, with a 30 -day mortality rate of $35-52 \%$, with $50 \%$ of the deaths occurring in the first 2 days [3-5]. After initial irreversible tissue injury is suffered near the hemorrhage nidus, a progressive cascade of elevated local pressure, edema, and excitotoxicity cause additional 
secondary injury to surrounding areas [6-8]. Secondary brain injury by hematoma often occurs in the days following the initial hemorrhage and is intimately associated with significant neurological deterioration.

Despite being considerably frequent, the treatment of hematomas within the basal ganglia continues to be a matter of debate among neurologists and neurosurgeons. The present powerful evidence provided by the results of the International Study of the Treatment of Intracranial Hemorrhage (STICH) corroborates this statement which has been established previously: there was not significant benefit for conventional aggressive surgical treatment over conservative medical treatment for the acute care of ICH [9]. Nevertheless, more than 7,000 patients with ICH in the United States undergo traditional evacuation procedures each year [10].

However, various clinical studies testing the hypothesis that clot burden plays a significant role in several forms of intracranial hemorrhage have been published in recent years, suggesting that clot reduction plays an important role in limiting brain edema and additional neuronal injury, as well as in reducing the severity of neurological deficits following ICH [11-14]. Because of the lack of validated therapeutic options for this form of stroke, the role of minimally invasive surgery (MIS) in the treatment of ICH has gained importance and several different operational methods have emerged over the past decade. In this context, our treatment with a stereotactic technique, which we have termed minimally invasive stereotactic puncture and thrombolysis therapy (MISPTT), is herewith presented.

MISPTT is a novel operative technique for $\mathrm{ICH}$, which was developed by Pro Jia in 1997. Although several clinical studies on MISPTT in the acute phase of ICH have been published during the past decade, the impact of MISPTT on the short-term and long-term neurological function of patients who survive the acute phase is less clear. The purpose of the present study was to investigate whether the long-term and short-term benefits of MISPTT are maintained and whether this method improves the ultimate outcome in these ICH patients. Therefore, we compared the long-term outcome 1 year after treatment in a consecutive series of ICH patients treated by MISPTT with the results achieved in a comparable group of patients who were treated by conventional craniotomy (CC).

\section{Methods}

A randomized control clinical trial was undertaken. All ICH patients were inhospital patients from 2005-2008 diagnosed with $\mathrm{ICH}$, according to the $\mathrm{ICH}$ criteria drafted by ASA [15]. All cases were monitored in a dedicated stroke unit. The volume of the ICH in milliliters $(\mathrm{mL})$ was estimated on the basis of approximate ellipse volume with the formula $\mathrm{A} \times \mathrm{B} \times \mathrm{C} / 2$, where $\mathrm{A}$ represents the largest diameter of the hematoma on axial CT slices in centimeters, $\mathrm{B}$ the diameter of hematoma perpendicular to $\mathrm{A}$ on the same slice, and $\mathrm{C}$ the number of CT slices in which the hematoma is visible multiplied by the slice thickness in centimeters $[16,17]$.

\section{Inclusion and exclusion criteria for patients}

Inclusion criteria were as follows: (1) diagnosed as having spontaneous hemorrhage in the basal ganglion or brain lobe of the brain by CT scan; (2) hemorrhage volume: 30-100 ml; (3) age range: 40-75 years; (4) muscle strength of the paralyzed limbs: grades $0-3$ on the muscle strength scale; (5) hemorrhagic duration (from stroke onset to hospital) within $24 \mathrm{~h}$; and (6) informed consent from patients and/or their legal representative.

Exclusion criteria were as follows: (1) disturbances of blood coagulation, e.g., thrombocytopenia, hepatitis; (2) traumatic intracranial hemorrhage; (3) intracranial or general infection; (4) complicated with serious heart, liver, renal, or lung disease or functional failure; (5) a previous stroke history with neurological deficits; (6) intracranial aneurysm or arteriovenous malformation complicated with hemorrhage; (7) consent form not obtained from the patient or their legal representative.

There were 122 patients analyzed, of which 64 cases were treated with MISPTT and 58 cases with CC. There were no statistically significant differences in sex, age, GCS score, localization of bleeding, hemorrhage volume, or blood pressure level during hospitalization, duration of hypertension and the accompanying diseases in the two groups. Although the accompanying diseases such as diabetes, hyperlipidemia, coronary heart disease and cerebral infarction in the two groups were similar (all $P>0.05$ ), diabetes accounted for $62.5 \%$ and $60.3 \%$, respectively, in MISPTT group and CC group, and was perhaps one important risk factor for ICH onset (Table 1).

Treatment methods

Minimally invasive stereotactic puncture and thrombolysis therapy

All operations were performed under local anesthesia and intravenous sedation unless the patient was already intubated for medical or neurological indications independent of the procedure. Stereotactic aspiration of the hematomas was performed in the acute phase between the 6th and the 24th hour after onset of stroke as required in the MISPTT protocol. First, the target points were defined according to the $\mathrm{CT}$, and target points were generally chosen in the scan 
Table 1 The baseline characteristics of patients

\begin{tabular}{|c|c|c|c|}
\hline Group & MISPTT patients & CC patients & $P$ value \\
\hline Number of patients & 64 & 58 & \\
\hline Gender (m:f) & $41: 23$ & $38: 20$ & 0.866 \\
\hline Mean age (years) & $58.7 \pm 9.0$ & $57.6 \pm 8.6$ & 0.483 \\
\hline GCS score $(n / \%)$ & & & 0.998 \\
\hline $4-5$ & $7 / 10.9$ & $6 / 10.3$ & \\
\hline $6-9$ & $22 / 34.4$ & $20 / 34.5$ & \\
\hline $10-12$ & $23 / 35.9$ & $19 / 32.8$ & \\
\hline $13-15$ & $12 / 18.8$ & $13 / 22.4$ & \\
\hline Hematoma volume $(n / \%)$ & & & 0.987 \\
\hline 30-49 (mL) & $27 / 42.2$ & 22/37.9 & \\
\hline 50-79 (mL) & $23 / 35.9$ & $21 / 36.2$ & \\
\hline 80-100 (mL) & $14 / 21.9$ & $15 / 25.9$ & \\
\hline Direction of the hematoma $(n / \%)$ & & & 0.468 \\
\hline Left-sided & $34 / 53.3$ & $27 / 51.3$ & \\
\hline Right-sided & $30 / 46.7$ & $31 / 48.7$ & \\
\hline Location of the hematoma $(n / \%)$ & & & 0.344 \\
\hline Basal ganglia & $48 / 53.3$ & $39 / 50.0$ & \\
\hline Thalamus & $16 / 17.8$ & $19 / 24.4$ & \\
\hline \multicolumn{4}{|l|}{ Mean BP } \\
\hline SBP & $174.3 \pm 14.1$ & $172.2 \pm 11.0$ & 0.361 \\
\hline DBP & $96.7 \pm 9.7$ & $98.8 \pm 9.4$ & 0.255 \\
\hline Duration of BP (years) & $7.1 \pm 1.9$ & $7.4 \pm 2.0$ & 0.312 \\
\hline \multicolumn{4}{|l|}{ Accompanied disease $(n / \%)$} \\
\hline Diabetes & $40 / 62.5$ & $35 / 60.3$ & 0.807 \\
\hline Hyperlipidemia & $27 / 42.2$ & 22/37.9 & 0.632 \\
\hline Coronary heart disease & $20 / 31.3$ & $21 / 36.2$ & 0.563 \\
\hline Cerebral infarction & $14 / 21.9$ & $15 / 25.9$ & 0.605 \\
\hline
\end{tabular}

with the largest expansion of the hematoma and special attention was paid to stay away from important cortex function areas (Fig. 1). Puncture situs was measured and marked on the head, again avoiding main blood vessels, then a puncture needle of suitable length (type YL-1) was fixed to the operative electric drill. The puncture needle was entered to the predetermined depth, then the probe core was removed; the hematoma was withdrawn gently using a syringe (diluted with saline if blood thickened) until one-third of hematoma was removed, then a needlelike hematoma disintegrator was inserted. When no more blood could be withdrawn, the hematoma cavity was thoroughly rinsed with saline, until the saline fluid was clear.

To confirm gross hematoma evacuation, an immediate postoperative CT scan was taken to assess the puncture needle placement and residual hematoma volume. If the placement of puncture needle was at the center of hematoma and stable, without rebleeding, a drainage bag connected to the puncture needle was hung $10 \mathrm{~cm}$ upon the head and switched for drainage. Upon rebleeding, $1 \mathrm{ku}$ reptilase or $1 \mathrm{mg}$ adrenalin should be injected into the hematoma, drained after 0.5 hour, and rinsed after 6-8 hours. All patients were treated in a dedicated intensive care unit, where subsequent thrombolysis and clot drainage were performed at the bedside using sterile techniques. The hematoma was continuously liquefied by liquefacient (containing 20,000-40,000 U urokinase/ 2-3 mL saline solution) for 2-4 days (3-5 times/day).On the $1 \mathrm{st}$, 3rd, 5th, and 7 th day of postoperation, patients were re-examined using CT. For hematomas that are difficult to liquefy, it should be liquefied as far as possible at first, followed by repeated, gentle aspiration using an agitation maneuver of the liquefacient to achieve vacuity. For hematomas affecting one lateral cerebral ventricle, then only that hematoma cavity should be perforated, but for patients with a hematoma affecting both lateral cerebral ventricles and the casting mould, the hematoma cavity and the contralateral cerebral ventricle should be perforated simultaneously.

In addition, for those patients with a hematoma breaking into the lateral cerebral ventricle, lumbar puncture could be 


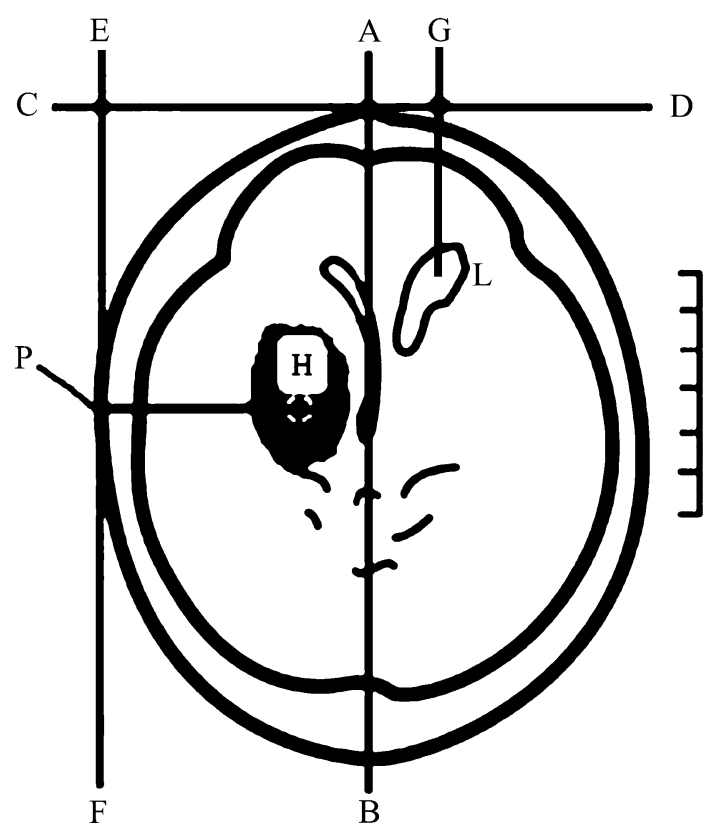

Fig. 1 The slice of the largest hematoma area and puncture point in the $C$ T are illustrated. $A B$ median sagittal line, $C D$ precoronal line, $E F$ lateral sagittal line, $P$ puncture point of hematoma, $H$ center of hematoma, $G$ puncture point of lateral cerebral ventricle, $P H$ puncture depth, $L$ lateral cerebral ventricle

performed to repeatedly replace cerebral spinal fluid using NS until the color is pale for 4-5 days (once/day). When the hematoma was cleared or the remaining volume was less than $10 \mathrm{~mL}$, the general condition of the patient was stable and intracranial pressure was normal; the puncture needle was removed, if no intracranial hypertension developed after occluding the drainage tube for 24 hours. The puncture needle was removed at the bedside under sterile conditions, and a single suture was placed at its exit site and covered with an occlusive dressing. In addition, all patients received optimal medical treatment.

\section{Conventional craniotomy}

Between 6 and 24 hours after onset, clearance of the hematoma by traditional craniotomy with large bone flap removal was performed in the Department of Neurosurgery. The medical treatment was routinely performed.

\section{Follow-up and outcome assessment}

Evaluation of all 122 patients followed identical criteria. Initial assessment included baseline characteristics (age, gender, HV, etc). The pretreatment clinical state of this cohort was assessed according to the GCS. All 122 cases in the two groups could be matched with regard to baseline characteristics. The posttreatment clinical state of the patients was assessed according to the GSC score, incidence of complications, and recurrence of bleeding after surgery. Outcome 1 year after stroke was the major endpoint. Four outcome parameters commonly used to assess outcome were employed to study the full impact of hemorrhagic stroke on long-term follow-up. Total case fatality was defined 1 year after stroke. According to the Glasgow outcome scale (GOS), clinical outcomes were graded, ranging from good recovery (GOS 5) to dead (GOS 1 ). The performance and functional status of the patients in activities of daily living (ADL) were measured with the Barthel index (BI 0-100). Disability was assessed using the modified Rankin scale (mRS 0-5). The data of all four outcome parameters for the two treatment groups were analyzed and compared.

\section{Statistics}

Categorical variables were analyzed using the $\chi^{2}$ test or Fisher's exact test for small samples. Measurement data were analyzed using $t$ tests. All recorded data were input using Epi Info software and statistically analyzed using SPSS 11.5 statistical software. For all analyses, $P<0.05$ was considered to be statistically significant.

\section{Results}

Comparison of consciousness level after surgery, GCS score, and the incidence of complications between the two groups

The GCS scores between the two groups prior to the operation were not significantly different $(P=0.998)$. The total incidence of complication in the MISPTT group was lower than that of the CC group $(P=0.000)$. Pulmonary infection, digestive tract hemorrhage, and epilepsy in the MISPTT group were all lower than that observed in the CC group $(P=0.047, P=0.016$, and $P=0.010$, respectively). The incidence of bleeding recurrence was not significantly different between the two groups $(P=0.199)$ (Table 2). Typical examples from the study are shown; for example, CT scans of one coma patient (GCS score 5) with a huge hematoma $(>70 \mathrm{~mL})$ are shown in Fig. 2. There were obvious difference in the $\mathrm{HV}$ and edema of surrounding brain before MISPTT with a GCS score of 5 and 2 weeks after MISPTT with a GCS score of 14 . The patient was self-sufficient 1 year after on-set (Fig. 2).

Comparison of long-term outcome and total case fatality of the two groups 1 year after stroke

There were no sharp differences in case fatality between the MISPTT group and the CC group 1 year after stroke 
Table 2 Consciousness level after surgery, GCS score, and incidence of complications

\begin{tabular}{llll}
\hline Group & MISPTT patients & CC patients & $P$ value \\
\hline Number of patients & 64 & 58 & \\
GCS score & & & \\
$\quad$ Before operation & $8.7 \pm 1.9$ & $8.4 \pm 2.2$ & 0.445 \\
After operation & $11.0 \pm 1.2$ & $8.1 \pm 1.0$ & 0.000 \\
Incidence of complication $(n / \%)$ & $20 / 31.3$ & $46 / 79.3$ & 0.000 \\
Pulmonary infection & $6 / 9.4$ & $13 / 22.4$ & 0.047 \\
Digestive tract hemorrhage & $10 / 15.6$ & $20 / 34.5$ & 0.016 \\
Epilepsy & $4 / 6.3$ & $13 / 22.4$ & 0.010 \\
Bleeding recurrence $(\mathrm{n} / \%)$ & $6 / 9.4$ & $10 / 17.2$ & 0.199 \\
\hline
\end{tabular}

Brain CT scans before MISPTT
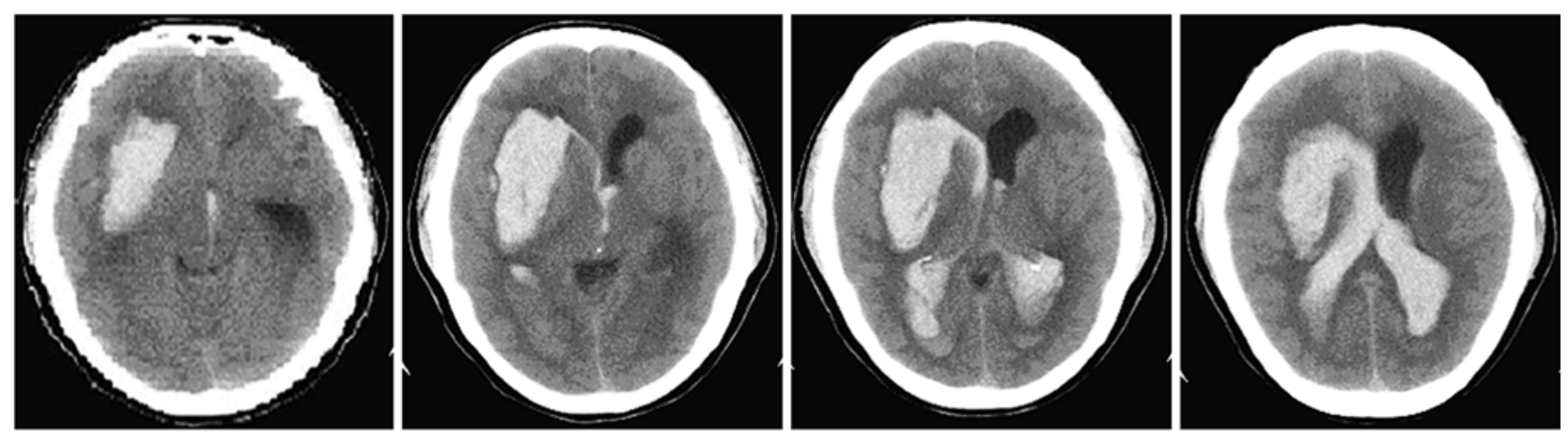

Brain CT scans 2 weeks after MISPTT
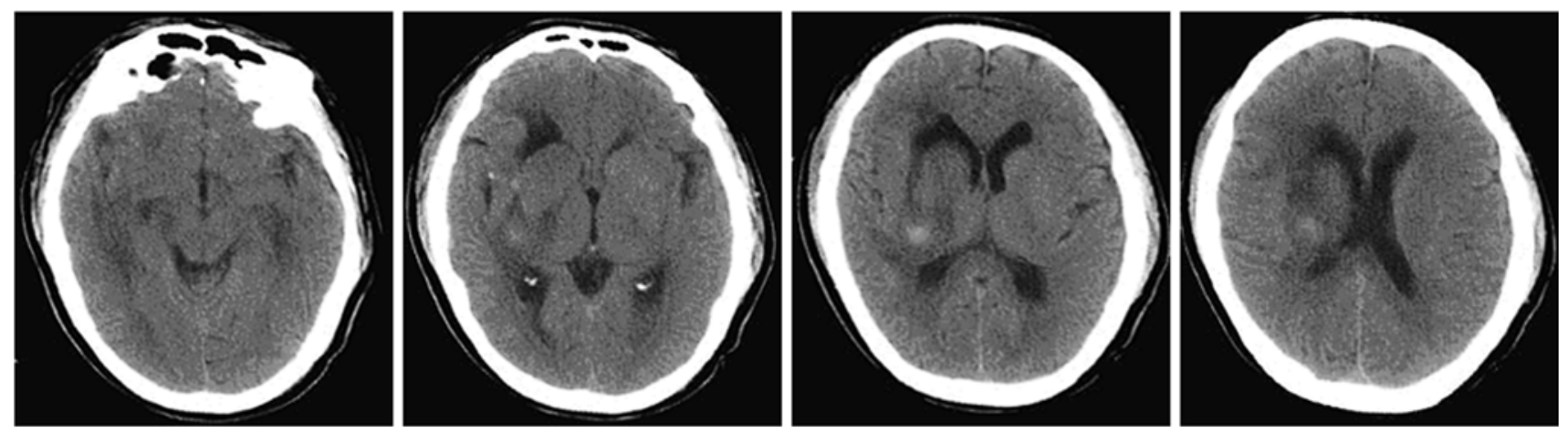

Fig. 2 CT scans of a patient in a coma (GCS score 5) with a large hematoma ( $>70 \mathrm{~mL}$ ) before MISPTT (top), and CT scans of the same patient during consciousness (GCS score 14) 2 weeks after MISPTT (bottom). These scans demonstrate the size of the hematoma on serial

(17.2 and $25.9 \%$, respectively, $P=0.243$ ); however, on the whole, the results showed that the survival in the MISPTT group was significantly better than that of the CC group. There were 27 cases with a $\mathrm{HV}<50 \mathrm{~mL}$ in the MISPTT group and there were no deaths 1 year postoperation, and 22 cases with a $\mathrm{HV}<50 \mathrm{~mL}$ in the CC group, of whom 2 had died within 1 year postoperation. Three parameters representing long-term outcome, the GOS $(4.4 \pm 0.6)$, BI $(82.6 \pm 9.5)$, and $\mathrm{mRS}(1.8 \pm 1.0)$ in the MISPTT group with a $\mathrm{HV}<50 \mathrm{~mL}$ had significantly axial CT images. CT scans 2 weeks after MISPTT showed obvious amelioration in hematoma volume and edema in the surrounding brain compared with that prior to MISPTT. The patient was able to completely return to a normal lie 1 year after on-set

ameliorated compared to those in the CG group (GOS $3.4 \pm 1.1$, BI $73.0 \pm 14.6$, and $\mathrm{mRS} 2.7 \pm 1.3$, respectively) $(P=0.000, P=0.009$, and $P=0.009$, respectively). There were 37 cases with a $\mathrm{HV} \geq 50 \mathrm{~mL}$ in the MISPTT group, of whom 11 patients had died 1 year postoperation, while there were 36 cases with a HV $\geq 50 \mathrm{~mL}$ in the CC group and 13 patients had died 1 year postoperation. The GOS ( $3.3 \pm 1.6)$, BI $(73.3 \pm 15.6)$, and $\mathrm{mRS}(2.2 \pm 1.2)$ in the MISPTT group with a HV $\geq 50 \mathrm{~mL}$ had significantly ameliorated compared to those in the CG 
Table 3 Outcome 1 year after stroke

GOS Glasgow outcome scale, $m R S$ modified Rankin Scale, $B I$ Barthel index, $H V$ Hematoma volume

\begin{tabular}{llll}
\hline Group & MISPTT patients & CC patients & $P$ value \\
\hline $\begin{array}{l}\text { Number of patients } \\
\text { Case fatality }(n / \%)\end{array}$ & 64 & 58 & \\
GOS & $11 / 17.2$ & $15 / 25.9$ & 0.243 \\
$\mathrm{HV}<50 \mathrm{~mL}$ & $4.4 \pm 0.6(27$ cases $)$ & $3.4 \pm 1.1(22$ cases $)$ & 0.000 \\
$\mathrm{HV} \geq 50 \mathrm{~mL}$ & $3.3 \pm 1.6(37$ cases $)$ & $2.5 \pm 1.5(36$ cases $)$ & 0.040 \\
$\mathrm{BI}$ & & & \\
$\mathrm{HV}<50 \mathrm{~mL}$ & $82.6 \pm 9.5(27$ cases $)$ & $73.0 \pm 14.6(20$ cases $)$ & 0.009 \\
$\mathrm{HV} \geq 50 \mathrm{~mL}$ & $73.3 \pm 15.6(26$ cases $)$ & $62.2 \pm 21.5(23$ cases $)$ & 0.042 \\
$\mathrm{mRS}$ & & & \\
$\mathrm{HV}<50 \mathrm{~mL}$ & $1.8 \pm 1.0(27$ cases $)$ & $2.7 \pm 1.3(20$ cases $)$ & 0.009 \\
$\mathrm{HV} \geq 50 \mathrm{~mL}$ & $2.2 \pm 1.2(26$ cases $)$ & $3.0 \pm 1.6(23$ cases $)$ & 0.033 \\
\hline
\end{tabular}

group (GOS $2.5 \pm 1.5, \quad \mathrm{BI} \quad 62.2 \pm 21.5$, and $\mathrm{mRS}$ $3.0 \pm 1.6$, respectively $)(P=0.040, \quad P=0.042$, and $P=0.033$, respectively). The results are shown in Table 3 .

\section{Discussion}

The mass effect of a hematoma can lead to the brain damage, including intracranial hypertension or cerebral hernia [18]. There is, however, some evidence that the mass effect caused by a $\mathrm{HV}(<60 \mathrm{~mL})$ was not the dominant injury mechanism, whereas the toxic substances released from the hematoma were the most important factor in the pathological mechanism of the cerebral hemorrhage [19-21]. It was reported that elevated levels of glutamate were found in the perihematomal region after ICH and these levels decreased after hematoma drainage. Conversely, ischemic LPRs were not found in perihematomal regions and were unchanged after hematoma drainage. These data suggest that excitotoxicity related to glutamate may have an important impact on secondary injury. The data failed to support the role of ischemia in secondary perihematomal damage [6].

Thus, effective removal of the hematoma during the acute phase is a crucial principle in the treatment of ICH in order to save lives and improve long-term quality of life. $\mathrm{CC}$ by removing a bone flap is a classical technique for the treatment of ICH, which is characterized by good visualization, complete clearance of the hematoma, easy hemostasia, and complete reduction of pressure, but also has some shortcomings such as length of operation, severe brain damage due to manipulation of the brain during the operation, damage of brain tissue around the hematoma by electrocoagulation, re-bleeding readily, pathophysiological changes postoperation (e.g., disturbance of water and electrolyte balances, fluctuation of blood sugar, instability of life signs), which result in severe impairment of neurological function, multiple complications, higher invalidism rates and fatality rates. With regard to basal ganglia hemorrhages, comparing the outcome of patients treated surgically with that of patients managed conservatively, many earlier and current publications showed no benefit from conventional surgery $[9,10,22-25]$. Only a few reports have demonstrated a trend towards better outcomes with conventional surgery [26-28]. While analyzing the given data, it becomes obvious that the major drawback in all these studies is the heterogeneity of the ICH patient groups with regard to their preoperative neurological status due to the very different degrees of neurological impairment and no uniform consciousness levels, applying different surgical approaches, and different intervals with regard to the onset of the hemorrhagic stroke. Thus, it is an essential issue to select appropriate patients and a homogenous group to determine whether patients truly benefit from the neurosurgical stereotactic evacuation of the hematomas in the acute phase. In many recent studies, the minimally invasive methods have shown to be highly efficient with little risk of re-bleeding and better short-term outcomes [29-31]. Presently, some clinicians are exploring new methods to elevate curative effect of minimally invasive operation techniques. A study by Marquardt and coworkers [32] focused on the use of a novel multiple target aspiration technique in 64 patients to aspirate a "sufficient proportion" of the hematoma with minimal risk for the patient. More than $80 \%$ of the hematoma volume was successfully aspirated in $73.4 \%$ of the patients with only one episode of re-bleeding. Montes et al. [33] showed that CT-guided thrombolysis and aspiration was safe and effective in the reduction of $\mathrm{ICH}$ volume. In the meantime, they proposed further studies were needed to assess optimal thrombolytic dosage, including controlled comparisons of mortality, disability outcome, time until convalescence, and cost of care in treated and untreated patients.

MISPTT is a new and novel operative technique that is obviously different from other types of minimally invasive operations in design principle, which was developed by Pro 
Jia in 1997 with a distinctive thrombolysis installation, and highly safe and efficient function for dissolving and draining coagulated blood. This treatment had been widely applied in China. According to some studies in the past, it was presented that MISPTT in acute ICH efficiently cleared the hematoma, relieved hydrocephalus, reduced intracranial hypertension, and relieved the cytotoxicity of blood thrombin. Furthermore, the washing liquor decreased the levels of cytotoxic substances. In subacute hemorrhage, MISPTT reduced the neurotoxicity of the hemoglobin and its decomposition products. This technique is characterized by its simplicity and is not limited by equipment. Puncturing the brain in MISPTT does little harm to the brain and accelerates recovery of cerebral function, while the liquefaction technique contributes to liquefying the blood coagulum-all helping to shorten the course of disease. In the operations performed, patients were only treated with a $3 \mathrm{~mm}$ needle (diameter). Because there is no gap between the needle and skull, the incidence of infection is reduced. Furthermore, it is not necessary to open the skull and use general anesthesia; thus, this procedure is more economical than other operations. The Chinese National Research and Extension Community of the Minimally Invasive Operation suggested that MISPTT is suitable for cases having a hemorrhage volume $>30 \mathrm{~mL}$ in the basal ganglia and further standardized the operation indication, operation procedure, and the methods to apply the hematoma liquefacient, according to the random sampling of The Ministry of Public Health.

Although there are many studies investigating the minimally invasive operation indication for $\mathrm{ICH}$, few specially concerned the indication for the MISPTT. Furthermore, most of these investigations lacked control groups with uniform baseline characteristics prior to operation, did not analyze the correlation factors (e.g., GCS score, hematoma volume, hematoma location, duration of BP and accompanied diseases), and did not observe the long-term outcome for survival. In our study, the above shortcomings were overcome by the inclusion of many factors, e.g., preoperative neurological status, surgical approach, patient selection, GCS score, incidence of complications, rebleeding after surgery, and long-term outcome 1 year after onset. In addition, accompanying diseases in the two groups of ICH patients were observed, such as diabetes, hyperlipidemia, coronary heart disease and cerebral infarction. There were no statistically significant differences between the two groups (all $P>0.05$ ), but it was noticed that diabetes accounted for $62.5 \%$ and $60.3 \%$, respectively, in $\mathrm{MG}$ and $\mathrm{CG}$, which indicated that diabetes was perhaps one important risk factor for ICH onset (Table 1). The results showed that the level of consciousness and GCS in MISPTT were better than that of the CC group. Of the 13 patients with GCS scores of 4-5 in the two groups,
11 patients died $(84.6 \%)$; the two survivors were treated with MISPTT (Table 1, one case referring to Fig. 2). The incidence of complications (e.g., pulmonary infection, hemorrhage of the digestive tract, and epilepsy) in MISPTT was obviously reduced compare to the CC group. There were no cases of intracranial infection in either the MISPTT or the craniotomy group. The statistical analysis did not show a significant difference on the rebleeding incidence in the two groups (9.4 and $17.2 \%$, respectively, $P=0.199$ ). There were no obvious differences between the case fatalities in the MISPTT group and the CC group (17.2 and $25.9 \%$, respectively, $P=0.243$ ). The long-term outcome in the MISPTT group surpassed that of the CC group, with respect to GOS, mRS, and BI (all $P=0.000$ ).

Currently, the thrombolysis methods have only been partly explored. We used UK as thrombolysis method in our MISPTT study. In another cohort of ICH patients treated using FAST, volumetric analysis of ICH and perihematomal edema seemed to suggest that local use of rtPA for thrombolysis, which differed from the UK used in our MISPTT study, does not exacerbate brain edema formation. Furthermore, there seems to be a strong association between reduction of the $\mathrm{ICH}$ volume and reduction of edema volume, as would be expected following the concept of "hemotoxicity" postulated by some investigators [34].

These above results indicate that for the $\mathrm{ICH}$ patient meeting MISPTT operation requirements, it is unsuitable to choose $\mathrm{CC}$. However, if the patient has a huge hemorrhage volume, is rapidly deteriorating, or is in the early state of cerebral hernia, then a craniotomy should be selected to reduce cerebral pressure. In addition, another study [33] pointed out that although MIS on patients with cerebral hernia may not result in a good curative effect, the hematoma volume can be partially decrease and the intracranial pressure can be rapidly reduce, thus, gaining time for craniotomy. Of course, $\mathrm{CC}$ is occasionally superior in the treatment of ICH with bulk volume. Murthy et al. [35] combined decompressive craniectomy and hematoma clearance to cure 12 patients with hemorrhage volumes $>60 \mathrm{~mL}$ in the right hemisphere. As a result, 11 survivors were able to leave the hospital ( $92 \%$ survival rate), while in 6 cases, the survivors recovered well.

\section{Conclusion}

Larger randomized trials proving the increased benefits of MISPTT treatment over traditional craniotomy or purely medical management are currently in process. However, our study and some other treatment trials regarding this treatment modality have reported optimistic results, showing superiority of MMISPTT over CC or PMT. Several methodological issues surrounding this form of 
treatment remain to be resolved, including comparison of the relative efficacies of various mechanisms of clot thrombolysis and drainage. If successful, MISPTT for ICH may perhaps become an important tool in the growing treatment armamentarium for $\mathrm{ICH}$ and the potential for a disease modifying impact in these ICH patients. It is our hope that this ongoing study will bring us closer to other randomized tests regarding more relevant factors with MISPTT as an alternative treatment for ICH. Objectively, MISPTT is currently limited in its ability to achieve hemostasis and completely evacuate the hematoma. Proper selection of the ICH patient group to receive this type of therapy may hold the key to these advances.

Acknowledgments This work was supported by grants from the Major State Research Development Program of China (973 Program) (No. 2005CB522600).

\section{Conflicts of interest None.}

Open Access This article is distributed under the terms of the Creative Commons Attribution Noncommercial License which permits any noncommercial use, distribution, and reproduction in any medium, provided the original author(s) and source are credited.

\section{References}

1. Fayad PB, Awad IA (1998) Surgery for intracerebral hemorrhage. Neurology 51(3):S69-S73

2. Flaherty ML, Haverbusch M, Sekar P (2006) Long-term mortality after intracerebral hemorrhage. Neurology 66(8):1182-1186

3. Broderick JP, Brott T, Tomsick T (1993) Intracerebral hemorrhage more than twice as common as subarachnoid hemorrhage. J Neurosurg 78(2):188-191

4. Anderson CS, Chakera TM, Stewart-Wynne EG (1994) Spectrum of primary intracerebral hemorrhage in Perth, Western Australia, 1989-90-incidence and outcome. J Neurol Neurosurg Psychiatry 57(8):936-940

5. Carl C, Boonyakarnkul S, Dennis M (1995) Primary intracerebral haemorrhage in the Oxfordshire Community Stroke Project. Cerebrovasc Dis 5(1):26-34

6. Miller CM, Vespa PM, McArthur DL (2007) Frameless stereotactic aspiration and thrombolysis of deep intracerebral hemorrhage is associated with reduced levels of extracellular cerebral glutamate and unchanged lactate pyruvate ratios. Neurocrit Care 6(1):22-29

7. Wu JM, Hua Y, Keep RF (2003) Iron and iron-handling proteins in the brain after intracerebral hemorrhage. Stroke 34(12):29642969

8. Xi GH, Keep RF, Hoff JT (2002) Pathophysiology of brain edema formation. Neurosurg Clin N Am 13(3):371-383

9. Mendelow AD, Gregson BA, Fernandes HM (2005) Early surgery versus initial conservative treatment in patients with spontaneous supratentorial intracerebral haematomas in the International Surgical Trial in Intracerebral Haemorrhage (STICH): a randomised trial. Lancet 365(9457):387-397

10. Juvela S, Heiskanen O, Poranen A (1989) The treatment of spontaneous intracerebral hemorrhage: a prospective randomized trial of surgical and conservative treatment. J Neurosurg 70(5): $755-758$
11. Wagner KR, Xi GH, Hua Y (1999) Ultra-early clot aspiration after lysis with tissue plasminogen activator in a porcine model of intracerebral hemorrhage: edema reduction and blood-brain barrier protection. J Neurosurg 90(3):491-498

12. Teernstra OPM, Evers SMAA, Lodder J (2003) Stereotactic treatment of intracerebral hematoma by means of a plasminogen activator-a multicenter randomized controlled trial (SICHPA). Stroke 34(4):968-974

13. Vespa P, McArthur D, Miller C (2005) Frameless stereotactic aspiration and thrombolysis of deep intracerebral hemorrhage is associated with reduction of hemorrhage volume and neurological improvement. Neurocrit Care 2(3):274-281

14. Miller DW, Barnett GH, Kormos DW (1993) Stereotactically guided thrombolysis of deep cerebral hemorrhage: preliminary results. Cleve Clin J Med 60(4):321-324

15. Broderick J, Connolly S, Feldmann E (2007) Guidelines for the management of spontaneous intracerebral Hemorrhage in adults-2007 update - a guideline from the American Heart Association/American Stroke Association Stroke Council, high blood pressure research council, and the quality of care and outcomes in research interdisciplinary working group-The American Academy of Neurology affirms the value of this guideline as an educational tool for neurologists. Stroke 38(6):2001-2032

16. Brott T, Broderick J, Kothari R (1997) Early growth in patients with intracerebral hemorrhage. Stroke 28(1):1-5

17. Kothari U, Brott T, Broderick JP (1996) The ABCs of measuring intracerebral hemorrhage volumes. Stroke 27(8):1304-1305

18. Belayev L, Saul I, Curbelo K (2003) Experimental intracerebral hemorrhage in the mouse-Histological, behavioral, and hemodynamic characterization of a double-injection model. Stroke 34(9):2221-2227

19. Rabinstein AA, Atkinson JL, Wijdicks EFM (2002) Emergency craniotomy in patients worsening due to expanded cerebral hematoma-to what purpose? Neurology 58(9):1367-1372

20. Huang FP, Xi GH, Keep RF (2002) Brain edema after experimental intracerebral hemorrhage: role of hemoglobin degradation products. J Neurosurg 96(2):287-293

21. Georgiadis P, Xu HM, Chua C (2008) Characterization of acute brain injuries and neurobehavioral profiles in a rabbit model of germinal matrix hemorrhage. Stroke 39(12):3378-3388

22. Sykora M, Diedler J, Juttler E (2010) Intensive care management of acute stroke: surgical treatment. INT J Stroke 5(3):170-177

23. McKissock W, Taylor J, Richardson A (1961) Primary intracerebral hemorrhage: a controlled trial of surgical and conservative treatment in 180 unselected cases. Lancet 2(719):221-226

24. Elliott J, Smith M (2010) The acute management of intracerebral hemorrhage: a clinical review. Anesth Analg 110(5):1419-1427

25. Tan SH, Ng PY, Yeo TT (2001) Hypertensive basal ganglia hemorrhage: a prospective study comparing surgical and nonsurgical management. Surg Neurol 56(5):287-292

26. Hankey GJ, Hon C (1997) Surgery for primary intracerebral hemorrhage: is it safe and effective? A systematic review of case series and randomized trials. Stroke 28:2126-2132

27. Peng SY, Chuang YC, Kang TW (2010) Random forest can predict 30-day mortality of spontaneous intracerebral hemorrhage with remarkable discrimination. Eur J Neurol 17(7):945-950

28. Rincon F, Mayer SA (2010) Intracerebral hemorrhage: getting ready for effective treatments. Curr Opin Neurol 23(1):59-64

29. Barrett RJ, Hussain R, Coplin WM (2005) Frameless stereotactic aspiration and thrombolysis of spontaneous intracerebral hemorrhage. Neurocrit Care 3(3):237-245

30. Marquardt G, Wolff R, Janzen RWC (2005) Basal ganglia haematomas in non-comatose patients: subacute stereotactic aspiration improves long-term outcome in comparison to purely medical treatment. Neurosurg Rev 28(1):64-69 
31. Marquardt G, Wolff R, Sager A (2003) Subacute stereotactic aspiration of hematomas within the basal ganglia reduces occurrence of complications in the course of hemorrhagic stroke in non-comatose patients. Cerebrovasc Dis 15(4):252-257

32. Marquardt G, Wolff R, Seifert V (2003) Multiple target aspiration technique for subacute stereotactic aspiration of hematomas within the basal ganglia. Surg Neurol 60(1):8-13

33. Montes JM, Wong JH, Fayad PB (2000) Stereotactic computed tomographic-guided aspiration and thrombolysis of intracerebral hematoma: protocol and preliminary experience. Stroke 31(4): $834-840$

34. Carhuapoma JR, Barrett RJ, Keyl PM (2008) Stereotactic aspiration-thrombolysis of intracerebral hemorrhage and its impact on perihematoma brain edema. Neurocrit Care 8(3):322-329

35. Murthy JMK, Chowdary GVS, Murthy TVRK (2005) Decompressive craniectomy with clot evacuation in large hemispheric hypertensive intracerebral hemorrhage. Neurocrit Care 2(3):258-262 